\title{
En tiempos de pandemia: La enseñanza de lenguas extranjeras en la educación remota
}

\section{In times of pandemic: Foreign language teaching in remote education.}

\author{
Derly Cervantes Cerra ${ }^{1}$
}

Universidad del Atlántico, Colombia.

\section{RESUMEN}

El presente artículo de reflexión tiene como objetivo describir las herramientas TIC'S que se utilizan en la enseñanza de lenguas extranjeras en esta época de pandemia. Asimismo, se pretende responder a la premisa de si la enseñanza de lenguas extranjeras cambió a raíz de la pandemia que está afectando a la humanidad en general. En este sentido, en esta investigación documental, descriptiva e inscrita en el paradigma cualitativo, se realizó una revisión acerca de este tema y tuvo como principales hallazgos que las enseñanzas de lenguas extranjeras han utilizado desde hace muchísimas décadas las herramientas de las TIC'S y que el cambio de la modalidad de

1 Derly Cervantes Cerra

Orcid: 0000-0001-5386-2497

Dirección: Calle 19 \#22-24 Barrio Centro de Soledad

derly_cervantes@hotmail.com

Magíster en francés como Lengua Extranjera

Docente tiempo completo ocasional en el departamento de

Lenguas Extranjeras en la Universidad del Atlántico,

Colombia. educación presencial a remota, ha fortalecido las estrategias que se utilizaban tradicionalmente en la enseñanza de L2. Igualmente, se observó que otras asignaturas o áreas de aprendizaje, en la enseñanza remota, también empezaron a utilizar recursos que se emplean en la enseñanza de lenguas extranjeras como por ejemplo videos, audios, guías online interactivas, entre otros.

\section{PALABRAS CLAVE:}

enseñanza, lenguas extranjeras, pandemia, TIC'S.

\section{ABSTRACT}

The purpose of this reflection article is to describe the ICT tools used in foreign language teaching in this pandemic era. It also aims to answer the premise of whether the teaching of foreign languages has changed as a result of the pandemic that is affecting humanity in general. In this sense, in this documentary research, descriptive and inscribed in the qualitative 
paradigm, a review of this topic was carried out and the main findings were that foreign language teaching has been using ICT tools for many decades and that the change from faceto-face to remote education has strengthened the strategies that were traditionally used in L2 teaching. Likewise, it was observed that other subjects or learning areas, in remote teaching, also began to use resources used in the teaching of foreign languages such as videos, audios, interactive online guides, among others.

\section{KEYWORDS:}

teaching, foreign languages, pandemic, TIC'S.

Em tempos pandêmicos: Ensino de línguas estrangeiras na educação à distancia

\section{ABSTRATO}

Este artigo reflexivo visa descrever as ferramentas TIC utilizadas no ensino de línguas estrangeiras nesta época de pandemia. Ela também pretende responder à premissa de que o ensino de línguas estrangeiras mudou como resultado da pandemia que está afetando a humanidade em geral. Neste sentido, nesta pesquisa documental, descritiva e inscrita no paradigma qualitativo, foi feita uma revisão deste tema e as principais conclusões foram que o ensino de línguas estrangeiras tem usado ferramentas TIC por muitas décadas e que a mudança do ensino presencial para o ensino remoto fortaleceu as estratégias que eram tradicionalmente usadas no ensino L2. Da mesma forma, observou-se que outras disciplinas ou áreas de aprendizagem, no ensino à distância, também passaram a utilizar recursos que são utilizados no ensino de línguas estrangeiras, tais como vídeos, áudios, guias interativos on-line, entre outros.

\section{PALAVRAS-CHAVE:}

ensino, línguas estrangeiras, pandemia, TIC'S.

\section{INTRODUCCIÓN}

La palabra pandemia nunca había sido tan popular en los medios de comunicación e incluso charlas cotidianas, como lo es en la actualidad. El mundo entero convulsiona ante una de las enfermedades contagiosas más poderosas registradas: el covid-19. Desde finales de 2019 cuando medios internacionales como BBC Mundo, New York Times y otros, anunciaban las fatídicas complicaciones que estaba teniendo este virus en la nación nipona, la sociedad global ha ido cambiando de forma drástica la forma de interactuar entre las personas. Por ejemplo, ya no se puede salir a la calle sin una mascarilla, ya sea por protección propia o de los semejantes, tampoco está bien visto compartir una misma bebida entre varias personas, incluso el soplar las velas de un pastel de cumpleaños se considera prohibido por el alto riesgo de contagio que representa.

Ante este panorama, la educación cambió de forma imprevista y en algunas naciones, desordenadamente. Los docentes del mundo entero tuvieron que modificar sus clases presenciales a la nueva modalidad de aprendizaje remoto y esto puso en evidencia las distintas desigualdades sociales, curriculares e incluso tecnológicas que había entre distintos países y regiones del mundo (Sánchez, et All, 2020). $Y$ es que según la UNESCO citada en Villafuerte (2020) más de 800 millones de niños y jóvenes en más de 110 países han tenido que ver como su sistema educativo pasó de ser presencial a remoto y en condiciones que no necesariamente fueron favorables para su aprendizaje

Asimismo, para autores como Cortes (2021) la transición de presencial al aprendizaje remoto ha traído consigo una carga no solo de trabajo extra para los docentes, sino que además hubo un gran aumento en los niveles de estrés laboral, esto se puede observar en el siguiente fragmento de su estudio: 
Una labor muy loable que implementaron los docentes fue el redoblar las horas de trabajo, las cuales incluían adaptar los contenidos de sus asignaturas presenciales al aprendizaje remoto de emergencia, el uso prolongado de plataformas virtuales, la asignación de tareas, la forma de evaluarlas y la entrega de resultados; todos estos aspectos que influyeron en el éxito de la encomienda derivaba en una tarea agotadora para todo el cuerpo docente, el desgaste físico y emocional fue mayor, lo cual genera un estado mental que traen consigo altas consecuencias psicológicas y emocionales (p. 9)

En otras palabras, la transición de presencial a remoto o aprendizaje a distancia, no ha sido una tarea fácil de sobrellevar para el gremio docente en general. Pero, centrándose en un área específica de la educación como lo es la enseñanza de lenguas extranjeras es importante revisar si la dinámica de la enseñanza- aprendizaje de L2 cambió o si hubo algún fortalecimiento de las estrategias y uso de recursos TIC'S por ejemplo, que ya se venían empleando desde hace varias décadas.

\section{LA ENSEÑANZA DE LENGUAS EXTRANJERAS EN TIEMPOS DE PANDEMIA}

Según autores como Pastran, Gil y Cervantes (2020) durante la pandemia la utilización de las TIC'S pasaron de ser un recurso secundario o de apoyo, a ser las herramientas principales en la enseñanza de materias en general. Pero, en el caso de las lenguas extranjeras se transformaron en verdaderos protagonistas de la enseñanza y el aprendizaje. Los docentes de lenguas emplearon plataformas como Youtube para promover competencias comunicativas desde la multimodalidad que se enfoca no solo en lo lingüístico sino también en lo visual y no verbal que también intervienen en la construcción discursiva. Esto lo hicieron con ayuda de videos que estimulaban competencias como la escucha, el habla y la comprensión lectora al mismo tiempo. Y que, además, podían ser compartidos y discutidos casi que en tiempo real por medio de app como WhatsApp.

Es importante destacar, que antes de la pandemia ya había autores señalando las virtudes de emplear recursos TIC'S para fomentar el aprendizaje autónomo del estudiante en procesos que permitían al docente ser un mediador, pero no el protagonista, motivando, además la autorregulación del aprendizaje en el estudiante entre estos autores destaca por ejemplo Cassany citado por Lima y Araujo (2018), Berns, et All (2017), Cervantes (2019), Gil (2019), entre otros.

Asimismo, para autores como Taci (2020) la pandemia trajo consigo nuevas oportunidades para la enseñanza de las lenguas extranjeras por medio de recursos TIC'S. Porque si bien antes de la pandemia se veía el uso de pantallas como recursos secundarios y se le daba más prioridad a la interacción docente-estudiante en persona, con la llegada del virus y las medidas de aislamiento la pantalla pasó a ser un recurso sumamente valioso.

En este orden de las ideas, Plataformas comerciales como OPENENGLISH O ENGLISHLIVE vieron como sus metodologías de enseñanza por medio del internet eran incorporadas a las clases de distintas entidades educativas. Tener una clase por Skype, Zoom, Meet y otras, se volvió algo sumamente común. Tener foros de discusión en Facebook, Twitter, Instagram, Genially y demás, pasaron de ser solo un espacio de recreación a ser ambiente virtuales de aprendizaje (Álvarez, et All, 2020; Cervantes, 2019; Contreras, fuentes y González, 2020; entre otros)

En este sentido, era de esperarse que toda esta revolución en la adaptación de las clases presenciales a la virtualidad trajera consigo nuevas formas de aprendizaje y de interacción. 
Quizás ya existían antes de la pandemia, pero, sin duda alguna, en estos tiempos se han fortalecido. Según autores como Giannini y Grant (2020) y Diez (2020) esta revolución no tomó por sorpresa a los docentes de lenguas extranjeras, porque ya conocían los beneficios del uso de actividades que promocionaban la autonomía del estudiante. Destacan, por ejemplo, el empleo de videos y audios en ejercicios que debían ser realizados por los educandos en sus casas y en donde el rol del docente se caracterizaba por centrarse en guiar al estudiante en su propio proceso de aprendizaje.

Ha sido tal el éxito de la enseñanza de lenguas extranjeras desde el aprendizaje remoto que Taci (2020) postula que "hay un gran número de educadores, principalmente de países bien desarrollados, que encuentran la enseñanza en casa o virtual no como una solución temporal sino como una innovación futura" (traducción propia) (p. 19). En otros términos, hay un gran grupo de docentes de naciones consideradas con altos niveles de calidad de vida, que ven el éxito de la educación remota como un fuerte indicio de que la educación debería ser impartida así: desde la distancia.

Pero, esta realidad no representa la realidad de otros países como los latinoamericanos en el que los gremios docentes han denunciado no contar con los recursos apropiados para llevar a cabo sus clases de forma eficiente. Se quejan, por ejemplo, del poco acceso al servicio del internet tanto para ellos como para sus estudiantes, de no contar con el entrenamiento y capacitación adecuada para utilizar las distintas plataformas y metodologías de la llamada ahora educación remota (Cortes, 2021; Álvarez et. All, 2020; Toro, 2020; Valderrama, 2020; entre otros)

\section{EL USO DE LAS TIC'S EN LA ENSEÑANZA DE LENGUAS EXTRANJERAS: FORTALECIENDO EL APRENDIZAJE FLEXIBLE Y COLABORATIVO}

Según autores como Ramírez y García (2017) el uso de recursos TIC'S flexibiliza la forma de enseñanza y la de aprendizaje. Porque no solo no ata el proceso de enseñanza a un lugar específico como un aula de clases, sino que, además le permite al estudiante realizar sus actividades y tareas en horarios más flexibles que se adapten a sus intereses y necesidades, evidencia de ello es el siguiente fragmento:

Poder aprender sin las limitaciones de espacio y desde diferentes dispositivos se convierte en un fenómeno de grandes posibilidades dado que aumenta las ventajas propias del aprendizaje flexible al romper aún más las barreras espaciotemporales. El impacto sobre las posibilidades de aprender a través de dispositivos móviles en cualesquiera de los niveles educativos, resulta hoy incuestionable (p. 3)

Esta perspectiva es apoyada por Toro (2020) quien explica que el "acercamiento al uso de TIC que redundará en mayores posibilidades de flexibilidad, pertinencia, innovación y generación de competencias." (p. 52). Para este autor, el uso de las TIC'S además de fomentar la flexibilización también promociona la innovación docente en el aula. A lo que Cervantes (2019) agrega:

pueden ser extremadamente versátiles en cuanto al contenido que permiten compartir, pero su verdadero potencial radica en la capacidad que tienen para facilitar situaciones comunicativas en tiempo real que permiten además de la adquisición y mejora del vocabulario, practicar la expresión y comprensión oral (p. 120). 
Es evidente que, para los autores señalados, las TIC'S poseen un sin número de ventajas tanto para el estudiante como para el docente. Porque además de demandar creatividad del docente también demanda atención y un compromiso real del estudiante con su proceso de aprendizaje. Destacándose así, que su "propósito es el potenciar la eficiencia del sistema educativo" (Gil, 2019, p. 2). Cuestión que ya ha sido expuesta por Cordeiro citado por Lima y Araujo (2018), cuando señala que existen distintas plataformas cuya tecnología facilita la enseñanza - aprendizaje en el aula de lenguas extranjeras desde la perspectiva del aprendizaje colaborativo, dinámico e innovador. Evidencia de esto:

Me encanta la herramienta Genially para crear contenidos interactivos y dinámicos, así como presentaciones interactivas. Con esta aplicación y un poco de práctica y tiempo, es posible crear contenidos y actividades muy variadas (...) Quizlet es una aplicación para crear listas de vocabulario, generar tarjetas de vocabulario, juegos de tipo test con el fin de ayudar en la práctica del léxico y sus significados. Kahoot, también es muy útil para realizar actividades de repaso de una manera más competitiva y gamificada, y tiene mucho éxito entre los estudiantes. Wordle, sirve para convertir un texto libre en una nube de palabras, puede servir para ordenar las lluvias de ideas, sintetizar un texto en algunas palabras clave, crear un cuento o una historia a partir de una nube de palabras... (p. s/n)

Es palpable, que para la autora la diversidad de recursos y actividades que se pueden desarrollar por medio de las tecnologías tiene innumerables beneficios para la enseñanza de lenguas a remoto o a distancia. Porque se puede crear un ambiente virtual de aprendizaje interactivo, dinámico e incluso, ameno y divertido. Lo que redunda en estudiantes motivados al aprendizaje. El tema de la motivación también se ha vuelto un elemento de suma importancia en la educación remota porque se requiere de una estimulación extra para que el estudiante que también está viviendo la pandemia desde su realidad, se sienta incentivado a seguir su trayectoria escolar con el mismo o incluso más entusiasmo que tenía en la modalidad presencial (García, 2017; Pérez y Pastran, 2019; Rodríguez, 2016; Torres y Yepes, 2018; Villafuerte-Holguín, et All, 2020, entre otros)

\section{CONCLUSIONES GENERALES}

A lo largo de la revisión de la literatura, se pudo constatar que el proceso de adaptación de las clases presenciales a las virtuales trajo consigo muchísimo estrés para los docentes, pero también muchas oportunidades de mejora y fortalecimiento de la praxis desde el uso de las tecnologías. Sin embargo, los autores parecen coincidir en que el uso de las TIC'S por ejemplo, no era un tema nuevo en el área de enseñanza de lenguas extranjeras y que los profesores de idiomas tuvieron mayores oportunidades y facilidades en la adaptación de sus clases presenciales a la virtualidad o aprendizaje remoto. Además, esta investigación encontró que la mayoría de los trabajos consultados mostraba las ventajas y beneficios para la enseñanza - aprendizaje de incorporar con un rol más protagónico las herramientas de las TIC'S en las clases remotas. Porque resultaban una excelente alternativa para estimular el desarrollo y adquisición de competencias comunicativas en lenguas extranjeras desde el enfoque de la multimodalidad. Sin embargo, también hubo evidencia de que muchos docentes en países latinoamericanos, incluso los de idiomas, tenían serias dificultades para adaptar sus clases a la virtualidad debido a la falta de recursos y servicios relacionados con el internet.

$Y$ es que cuando se trata de transformar un paradigma educativo presencial a uno a distancia hay factores que pueden ir más allá de las capacidades y competencias de los docentes 
como el hecho de no contar con el apoyo institucional en el entrenamiento y capacitación del uso de plataformas nuevas. A ello, habría que agregar, las carencias de muchos estudiantes que difícilmente pueden acceder a recursos como computadores, teléfonos inteligentes o tabletas.

Para concluir, se afirma que esta época de pandemia ha obligado a los gremios docentes a mirar no solo el presente de la educación sino el futuro desde el uso de las tecnologías, y si bien verdad que en el aula de lenguas extranjeras el empleo de las TIC'S ya era común, también lo es que es necesario de una mirada más detallada y meticulosa de estos aspectos en otras áreas y asignaturas.

\section{REFERENCIAS BIBLIOGRÁFICAS}

Álvarez, H., Arias, E., Bergamaschi, A., López, Á., Noli, A., Ortiz, M., Pérez, M., RiebleAubourg, S., Rivera, M., Scannone, R., Vásquez, M. y Viteri, A. (2020) La educación en tiempos del coronavirus: Los sistemas educativos de América Latina y el Caribe ante COVID-19. Banco Interamericano del Desarrollo. http://dx.doi.org/10.18235/0002337

Berns, A., Palomo-Duarte, M., Isla-Montes, J. L., Dodero, J. M., y Torre, P. (2017). Agenda colaborativa para el aprendizaje de idiomas: del papel al dispositivo móvil. RIED. Revista Iberoamericana de Educación a Distancia, 20(2).

Cervantes, D. (2019). Las redes sociales y el aprendizaje de la lengua extranjera. Revista Boletín Redipe, 8(11), 117-123. https://doi.org/10.36260/rbr.v8i11.855

Cortés, J. (2021). El estrés docente en tiempos de pandemia. Dilemas contemporáneos: educación, política y valores, 8(1). https://doi.org/10.46377/dilemas. v8i. 2560
Contreras, L. Fuentes, H. \& González, K. (2020). Transformación de la educación frente a la pandemia y la analítica de datos. Revista Boletín Redipe, 9(7), 91-99. https://doi.org/10.36260/rbr.v9i7.1021

Díez, P. (2020). Learning Styles and their Adaptability to Information and Communication Technologies in Foreign Language Teaching. EDUTECHreview. Revista Internacional De Tecnologías En La Educación, 7(1), 29-35. https://doi. org/10.37467/gka-revedutech.v7.2458

García, L. (2017). Educación a distancia y virtual: calidad, disrupción, aprendizajes adaptativo y móvil. RIED. Revista Iberoamericana de Educación a Distancia, 20(2).

Giannini, S. y Grant, S. (2020). Three ways to plan for equity during the coronavirus school closures. UNESCO, (UNESCO Assistant Director-General for Education and, -UNESCO-IIEP Director). https://gemreportunesco.wordpress. com/2020/03/25/three-ways-to-plan-forequity-during-the-coronavirus-schoolclosures/

Gil, N. (2019). Ambiente virtual de aprendizaje: beneficios y ventajas para enseñanza del francés como L2. Revista Boletín Redipe, 8(11), 91-99. https://doi. org/10.36260/rbr.v8i11.852

Lima, G. y Araújo, J. (2018) El uso de las redes sociales para el desarrollo de la enseñanza de ele: el investigador y el profesor. Un diálogo entre estos dos sujetos a través de la entrevista con Daniel Cassany y Dayane Cordeiro. Trabalhos em Lingüística Aplicada, 57(2),1274-1296. Doi: https://dx.doi.org /10.1590/010318138651882364651 
Pastran, M., Gil, N. y Cervantes, D. (2020). En tiempos de coronavirus: las TIC'S son una buena alternativa para la educación remota. Revista Boletín Redipe, 9(8), 158-165. https://doi.org/10.36260/rbr. v9i8.1048

Pérez, A. y Pastran, M. (2019) ¿Qué es escritura académica desde la percepción del estudiante?. Conferencia llevada a cabo en el III Congreso Internacional de Investigación Lingüística en Medellín, Antioquia.

Ramírez, M. y García F. (2017). Presentación. La integración efectiva del dispositivo móvil enlaeducación y en el aprendizaje. RIED. Revista Iberoamericana de Educación a Distancia, 20(2)

Rodríguez, N. (2016) ¿Las TIC como mediadoras en la enseñanza-aprendizaje de lenguas extranjeras? Opción, 32 (10). 569-588. Recuperada de https://www.redalyc.org/ articulo.oa?id=31048901031

Sánchez, M., Martínez, A., Torres, R., Agüero, M., Hernández, A., Benavidez, M., Rendón, V. \& Jaimes, C. (2020). Retos educativos durante la pandemia de COVID-19: una encuesta a profesores de la UNAM. Revista Digital Universitaria, 21(3), 1-24.

Taci, J. (2020) Enseñanza del inglés como lengua extranjera en tiempos de pandemia ", Revista Europea de Educación, Centro Europeo de Educación e Investigación Científica, 3 ,

Toro, D. (2020). Educación superior en Latinoamérica en una economía postCOVID. Revista de Educación Superior en América Latina, ESAL 8, 45-53.

Torres, M. y Yépez, D. (2018) Aprendizaje cooperativo y TIC y su impacto en la adquisición del idioma inglés. Revista mexicana de investigación educativa, 23(78), 861-882

Valderrama, N. (2020). ¿Qué estamos haciendo los docentes por estos días y en qué tipo de educación estamos incursionando? Comunicación presentada en Experiencias de práctica a distancia. Universidad de los Andes. http://ued.uniandes.edu.co/Difusión/ Aprenderyense...

Villafuerte, P. (2020). Educación en tiempos de pandemia: COVID-19 y equidad en el aprendizaje. Observatorio de Innovación Educativa, Tecnológico de Monterrey. https://observatorio.tec.mx/edu-news/ educacion-en-tiempos-de-pandemiacovid19

Villafuerte-Holguín, J. S., Bello Piguave, J. E., Pantaleón Cevallos, Y., \& Bermello Vidal, J. O. (2020). Rol de los docentes ante la crisis del COVID-19. Una mirada desde el enfoque humano. Revista Electrónica Formación y Calidad Educativa, 8(1), 134-150. 